\title{
Heat Shock Proteins and Protection Against Ischemic Injury
}

\author{
W.H. Dillmann* \\ Department of Medicine, Endocrinology, and Metabolism, University of California at San Diego, \\ La Jolla, CA
}

\begin{abstract}
Heat shock proteins present a complex family of proteins exerting chaperone-like activities that are classified according to their molecular weight. We especially explored protective functions of inducible heat shock protein 70 , the mitochondrial heat shock protein 60 and 10 , and the small heat shock proteins HSP27 and $\alpha \mathrm{B}$-crystallin against ischemic, reoxygenation-mediated injury using transgenic animals and hearts under in vivo conditions and in isolated cardiac myocyte-derived cells using adenoviral vectors. We noted with great interest that differential protective effects are exerted by specific hsps. For example, alpha-B-crystallin and constitutive hsp70 markedly protect microtubular structure in cardiac myocytes from ischemia-induced injury. Inducible hsp70, hsp60 and hsp10 when coexpressed, and hsp27 and $\alpha \mathrm{B}$-crystallin have an overall protective effect against ischemic injury as determined by the release of enzymes like creatine kinase and LDH. We did not note inflammatory or immune responses elicited by the expression of hsps in transgenic animals and cardiac myocytes. The specific cell types in which hsps are expressed may contribute to the protective effect of hsps versus their inflammatory and immunogenic effects when expressed in other cell types. Infect. Dis. Obstet. Gynecol. 7:55-57, 1999. @ 1999 Wiley-Liss, Inc.
\end{abstract}

$\mathrm{H}^{\mathrm{c} e t}$ eat shock proteins (hsps) form a diverse group of proteins that are classified according to their molecular weight. ${ }^{1}$ Most members of the hsp family exert a chaperone or chaperonin-like function inside the cell, allowing proteins in the crowded intercellular environment to reach their proper final folding state in an accelerated fashion and avoiding folding structures that are not productive and lead to protein denaturation. This chaperone function results in a protective effect of hsps against noxious insults like heat ischemia and exposure to toxic chemicals. Several investigators have indicated that hsps can also exert an inflammatory role and serve as antigenic targets for the immune system and, in this way, can be detrimen- tal in certain pathophysiological circumstances. In this paper, the beneficial and protective features of hsps will be highlighted. The hsps discussed in further detail are the inducible hsp70, the small hsps hsp27 in humans or hsp25 in rodents, and alpha-B-crystallin. In addition, the mitochondrial hsps hsp60 and hsp10 that form the chaperonin complex in mitochondria are also considered.

A description of the response of the inducible hsp70 to ischemia follows. We noted some time ago that ischemia of the dog heart leads to a marked increase in the messenger RNA and protein level for the inducible hsp70., ${ }^{2,3}$ These findings raised the question whether increased expression of hsp70 could lead to a protective effect against isch-

*Correspondence to: Wolfgang H. Dillmann, MD, Department of Medicine, Endocrinology, and Metabolism, University of California San Diego, La Jolla, CA 92093-0618. E-mail: wdillmann@ucsd.edu 
emic injury. We initially investigated this question by stably transfecting $\mathrm{H} 9 \mathrm{C} 2$ cells, leading to an H9C2-cell line which overexpressed the human inducible hsp70. We found a significant increase in resistance to an ischemic stress in the H9C2 cells overexpressing the inducible hsp $70 .{ }^{4}$

In subsequent studies, we wanted to determine if this protective effect could also be induced under in vivo conditions. We therefore generated transgenic mice in which the rat inducible hsp70 is driven by a human cytomegalovirus enhancer chicken actin promoter. ${ }^{5}$ In initial studies, we used an isolated perfused mouse heart model to investigate protective effects of increased expression of hsp70 against ischemic injury. Hearts were harvested from transgene-positive mice and transgene-negative litter mates and submitted to perfusion and subsequently to sero flow ischemia for 20 minutes and reflow in the Langendorf perfusion setup. Recovery of contractile function was determined, the release of creatine kinase was investigated, and, in addition, myocardial infarct size was quantitated by a tetrazolium stain that exhibits a red color when exposed to viable myocardial tissue and a pale colorless stain in ischemic myocardium. We found that in hearts obtained from transgenic mice, the infarct zone was decreased by $40 \% .^{5}$ In addition, contractile function at 30 minutes of reflow was doubled, and the efflux of creatine kinase was decreased by $50 \%$. These findings, therefore, clearly indicate that protection against ischemic injury occurred in this isolated perfused heart model.

In subsequent studies performed in collaboration with Dr. Wolfe at the University of California San Francisco, we determined infarct size in a mouse in vivo model. ${ }^{6}$ The left coronary artery was occluded for 30 minutes, followed by 120 minutes of reperfusion. Infarcts were set in anesthetized mice overexpressing the inducible hsp70 transgene and in control mice. Infarct size was then determined after sacrifice of the animals by staining with tetrazolium, and we found that the infarct size was decreased by $50 \%$ in hsp70-positive transgenic mice. Expression of the hsp70 transgene in a large number of cardiac myocytes in cell culture can also be achieved by using adenoviral vectors. The hsp70 transgene was cloned into the E1 region of the viral vector, and infection of myocytes resulted in a significant increase in inducible hsp70 levels in marked protective effects against simulated ischemia. $^{7}$

In subsequent studies brief episodes of myocardial ischemia were used, leading to so-called myocardial stunning. Myocardial stunning occurs under conditions in which brief periods of ischemia do not to lead to permanent myocyte necrosis but result in a significant decrease in myocardial function after the ischemia is relieved. ${ }^{8}$ For this purpose, we developed an in vivo mouse model in which regional epicardial strains could be measured as an indicator of ischemia-induced myocardial malfunction. In addition, isolated perfused hearts were used to determine the contractile recovery after 10 minutes of global ischemia. In both of these models, the in vivo mouse model and the isolated heart, we found that expression of hsp70 protected against postischemic myocardial dysfunction induced by brief ischemic episodes or myocardial stunning.

Related to the protective effect of hsp60 and hsp10, we performed studies in collaboration with Dr. Mestril. For these studies, the hsp60 and hsp10 were cloned either by themselves or in combination into a human adenovirus five vector. Isolated neonatal myocytes were infected with the virus and then submitted to simulated ischemia. We obtained the very interesting result that only the coexpression of hsp60 and hsp10 could mediate a protective effect against simulated ischemia. In contrast, if hsp60 by itself or hsp10 by itself were expressed, no protective function could be elicited.

The last set of results relate to the protective effect of small hsps, hsp27 and alpha-B-crystallin. The small hsps arose most likely from a primordial gene and have a similar structure containing three exons. ${ }^{9}$ These small hsps form large multimeric units, and proteins that are not in their final folding stage bind to the outside of this large oligomeric complex. To determine if increased expression of the small hsps could result in a protective effect, we cloned alpha-B-crystallin and, separately, hsp27 into an adenoviral vector. The cardiac myocyte submitted to simulated ischemia that were infected with the adenoviruses expressing specific hsps were then subsequently examined for resultant damage. We observed that both hsp27 and alphaB-crystallin led to a significant protective effect measured by release of creatine kinase or LDH in 
isolated myocytes submitted to simulated ischemia. ${ }^{10}$ To investigate the detailed mechanism of protection, we investigated the integrity of specific skeletal structures in cardiac myocytes infected with different hsps and submitted to simulated ischemia. Especially when microtubular integrity was determined by indirect immunofluorescence using confocal microscopy and image analysis, we noted a significantly better protection of microtubular integrity in myocytes overexpressing alphaB-crystallin. In contrast, when hsp27 was overexpressed, no protection of microtubular structure occurred. We also noted that the constitutive hsp70 provided for increased microtubular integrity, in contrast to the inducible hsp70, which had no protective effect on microtubular structures. ${ }^{11}$

Related to the general protective effect of hsp27, we noted with great interest that the phosphorylation status of hsp27 does not play a significant role for this function. For example, when the three phosphorylatible serines 15,78 , and 82 were mutated to alanine, the same protective effect was mediated by the phosphorylation mutant versus the wild type hsp27.

In summary members of the hsp family, the inducible hsp70, constitutive hsp70, hsp60 and hsp10, and the small hsps hsp27 and alpha-Bcrystallin have very significant protective effects against ischemic injury under in vivo conditions and in isolated cardiac myocytes. The detailed target for the protective effect is different for the different hsps. For example, related to cytoskeletal structures, the integrity of microtubules is well preserved by alpha-B-crystallin but not by hsp27. In addition, the inducible hsp 70 protects microtubule structure but not constitutive hsp27. It will therefore be of great interest to determine the detailed mechanism by which specific hsps exert their protective function. It will also be of interest to determine whether increased expression of hsps in transgenic animals is tolerated without evidence of an inflammatory or immune response. In the transgenic animals we used, no such effect was observed; however, we have targeted hsps largely to cardiac myocytes that are not a competent im- mune-presenting cell, and, in this way, we may not have noted a down-side of increased hsp expression.

\section{REFERENCES}

1. Welch WJ, Mizzen LA, Arrigo AP. Structure and function of mammalian stress proteins. In Pardue ML, Feramisco JR, Lindquist S (eds.): New York: Alan R. Liss, 1989, pp 187-202.

2. Dillmann WH, Mehta HB, Barrieux A, Guth BD, Neeley WE, Ross J. Ischemia of the dog heart induces the appearance of a cardiac mRNA coding for a protein with migration characteristics similar to heat shock/stress protein 71. Circ Res 1986;59:110-114.

3. Mehta HB, Popovich BK, Dillmann WH. Ischemia induces changes in the level of mRNAs coding for stress protein 71 and creatine kinases M. Circ Res 1988;63: 512-517.

4. Mestril R, Chi SH, Sayen MR, O'Reilly K, Dilmann WH. Expression of inducible stress protein 70 in rat heart myogenic cells confers protection against stimulated ischemia induced injury. J Clin Invest 1994;93: 759-767.

5. Marber MS, Mestril R, Chi SH, Sayen MR, Yellon DM, Dillmann WH. Overexpression of the rat inducible 70$\mathrm{kD}$ heat stress protein in a transgenic mouse increases the resistance of the heart to ischemic injury. J Clin Invest 1995;95:1446-1456.

6. Huter JJ, Mestril R, Tam EKW, Sievers RE, Dillmann WH, Wolfe CL. Overexpression of heat shock protein 72 in transgenic mice decreases infarct size in vivo. Circulation 1996;94:1408-1411.

7. Mestril R, Giordano RJ, Conde AG, Dillmann WH. Adenovirus mediated gene transfer of a heat shock protein 70 (hsp70i) protects against simulated ischemia. J Mol Cell Cardiol 1996;26:2351-2358.

8. Trost SU, Omens JH, Karlon WJ, et al. Protection against myocardial dysfunction after a brief ischemic period in transgenic mice expressing inducible HSP70. J Clin Invest 1998;101:855-862.

9. Wistow G. Domain structure and evolution in -crystallins and small heat-shock proteins. FEBS Lett 1985;181: $1-6$.

10. Martin JL, Mestril R, Hilal-Dandan R, Brunton LL, Dillmann WH. Small heat shock proteins and protection against ischemic injury in cardiac myocytes. Circulation 1997;96:4343-4348.

11. Bluhm WF, Martin JL, Mestril R, Dillmann WH. Specific heat shock proteins protect microtubules during simulated ischemia in cardiac myocytes. Am J Physiol 1998;275:H2243-H2249. 


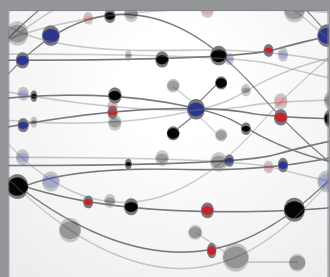

The Scientific World Journal
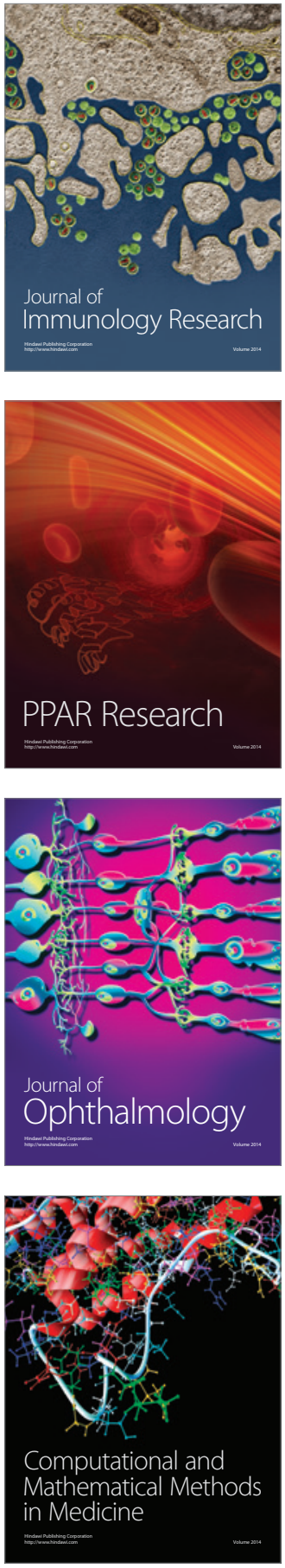

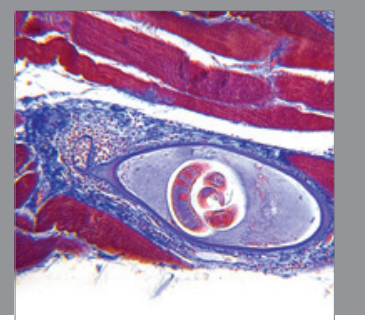

Gastroenterology

Research and Practice
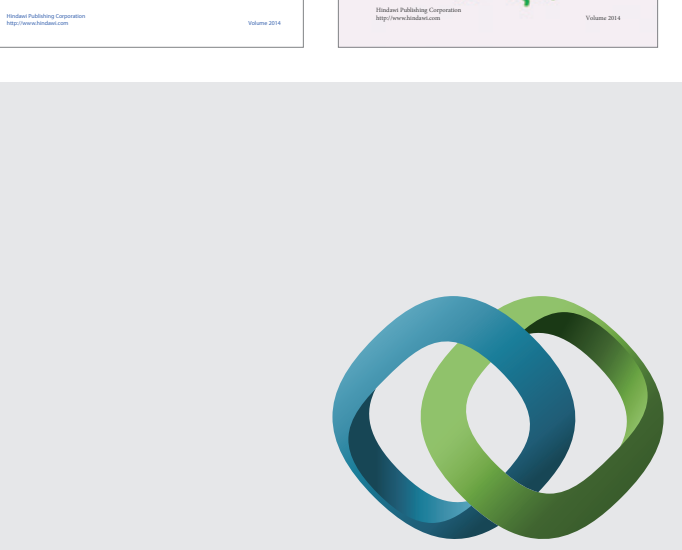

\section{Hindawi}

Submit your manuscripts at

http://www.hindawi.com
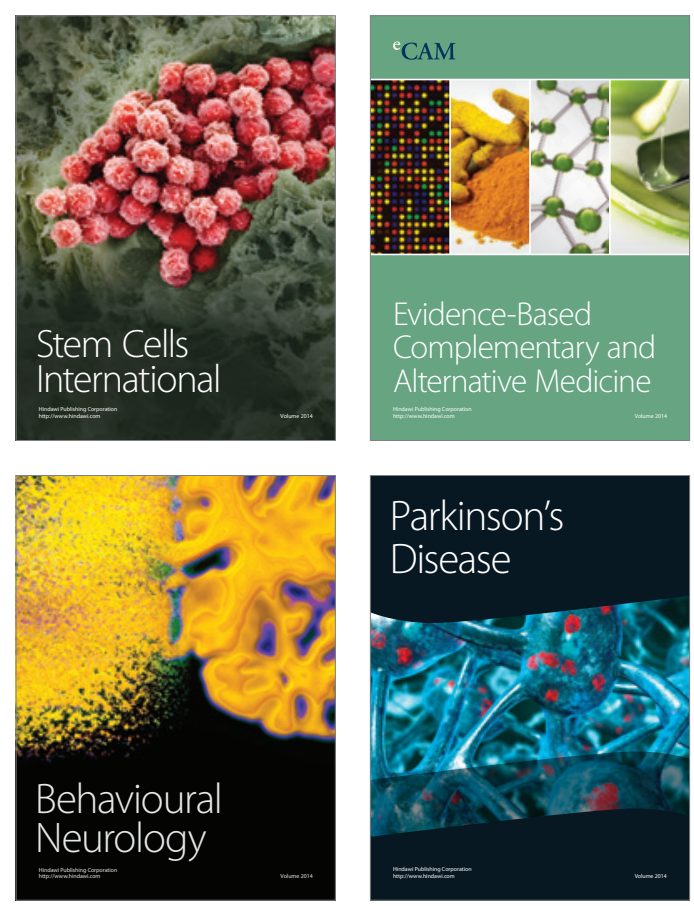

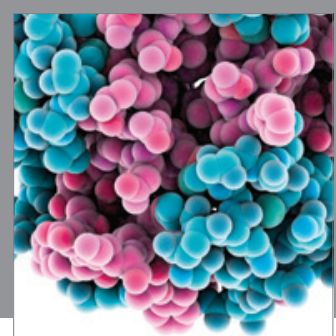

Journal of
Diabetes Research

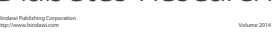

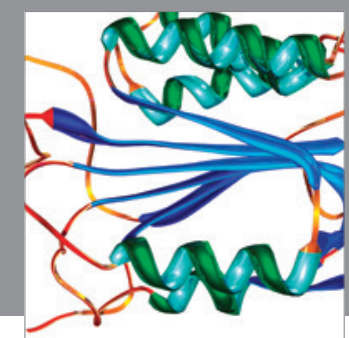

Disease Markers
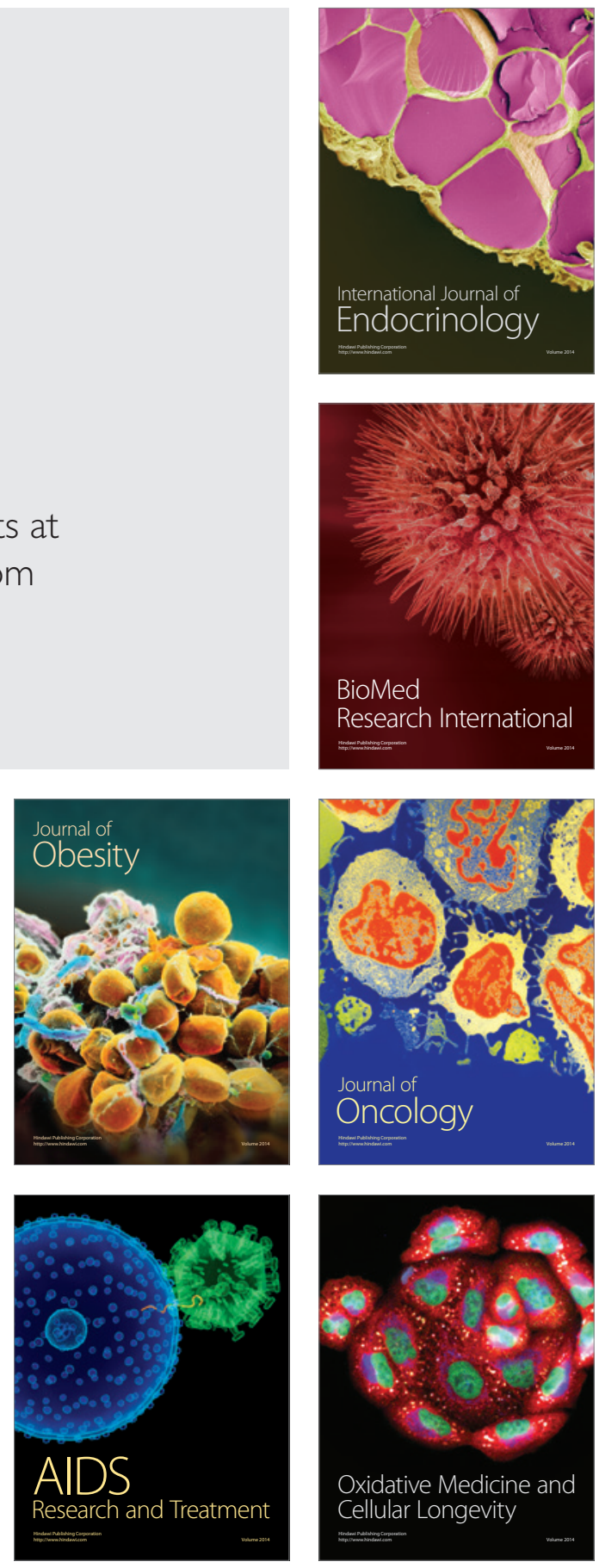